\title{
Ontologías del agua y relaciones de poder en torno al paisaje hídrico en el territorio indígena mazahua del estado de México
}

\author{
Water Ontologies and Power Relations around the Waterscape \\ in the Mazahua Indigenous Territory in México
}

\author{
Ariana Mendoza Fragoso* \\ Universidad Nacional Autónoma de México (UNAM), México
}

\begin{abstract}
RESUMEN
Este artículo aborda el paisaje hídrico como la forma en la que el agua es percibida e intervenida por diferentes grupos socioculturales. Entiendo el paisaje de manera relacional: desde una dimensión material reconozco los trazos que las prácticas de las sociedades imprimen en la naturaleza. Desde una dimensión ontológica, destaco la posibilidad y la política de una multiplicidad de mundos relacionados con el agua y de formas de estar con ella. A partir del caso de las comunidades indígenas mazahuas que habitan la sierra suroeste del estado de México -territorio de donde se trasvasa el $25 \%$ del agua que consume la Ciudad de México y su área metropolitana-, analizo el paisaje hídrico como un espacio de poder en donde se ensamblan distintas ontologías y prácticas políticas.
\end{abstract}

Palabras clave: paisaje hídrico, mazahuas, trasvase, poder, ontologías del agua.
DOI: https://doi.org/10.22380/2539472X.572

\section{ABSTRACT}

This article approaches the water landscape as the way in which water is perceived and intervened by different sociocultural groups. I conceive the landscape in a relational way: from a material dimension, which recognizes the traces that the practices of societies imprint on nature. And from an ontological one, that includes water representations, values, and epistemologies of different social actors. I analyze the waterscape as the space of power in which different ontologies and political practices are assembled, based on the case of the Mazahua indigenous communities that inhabit the southwest mountains of the State of Mexico, a territory from where $25 \%$ of the water consumed by Mexico City and its metropolitan area is transferred.

Keywords: waterscape, Mazahuas, hydraulic infrastructure, power, water ontologies.

Licenciada en Desarrollo y Gestión Interculturales de la UNAM y maestra en Gestión Sustentable del Agua. Actualmente, doctorante en Antropología Social en el Centro de Investigación y Estudios Superiores en Antropología Social, Ciudad de México. Su última publicación es: "Globalización, neoliberalismo e imperativos de la racionalidad económica en el uso de los recursos hídricos en México", en Ra Ximhai, número especial (enero-abril de 2013): 201-209, con Mazabel-Domínguez y Felipe-Macías. ary.xepje@gmail.com. 


\section{Introducción ${ }^{1}$}

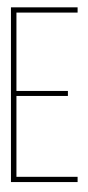

l agua, en tanto elemento primordial para los procesos de acumulación de capital, se convierte en un "lubricante" crucial del proceso de desarrollo económico (Swyngedouw 2004). Esto hace del agua un recurso estratégico que los grupos económicos poderosos se esfuerzan en controlar. Frente a los procesos de modernización, industrialización y urbanización — que implican una demanda creciente del líquido-, las regiones boscosas que captan el agua y la resguardan entre sus suelos y su vegetación se han convertido en blanco de proyectos de urbanización y extracción.

El despojo de tierras, el desplazamiento de poblaciones, las malas indemnizaciones y los bajos pagos por los terrenos representan solo una de las caras que han traído consigo el acecho y la especulación sobre estos territorios —principalmente indígenas y campesinos en el caso de México- (Gómez-Fuentes 2009). Otra cara de este proceso revela que, más allá de las negociaciones de compraventa de tierras o de proyectos compensatorios de desarrollo local, están en juego modos de vida ligados al agua, a las montañas y los bosques, que comprometen ontologías de gran importancia para el mantenimiento de esos territorios.

Tal es el caso del territorio indígena mazahua, enclavado en la sierra suroeste que rodea al Valle de Toluca, en la entidad federativa que lleva el nombre de estado de México (Edomex), de donde se trasvasa el agua del Sistema Cutzamala que abastece en promedio 20.000 litros de agua por segundo a la Ciudad de México y partes de su área metropolitana. Allí, entre las montañas y humedales, un modo de vida indígena ha habitado e interpretado un paisaje hídrico desde su propio contexto sociocultural. En ese lugar también se han producido conocimientos en torno a la naturaleza y sus ciclos. Continuamente se reconfiguran representaciones sociales que, a manera de mitos e imaginarios sobre el agua y las montañas, explican la dialéctica naturaleza-cultura, y además se fraguan y resignifican prácticas productivas, organizativas y rituales.

Este artículo quiere mostrar que así como el agua es un "recurso estratégico" para el desarrollo económico en la racionalidad dominante, también es valiosa en tanto es constitutiva de ontologías y epistemologías indígenas. El argumento que sostengo es que el trasvase de agua del Sistema Cutzamala, además de las injusticias económicas y ambientales que ocasiona, paulatinamente

Este artículo presenta algunos de los resultados de la investigación realizada entre el 2013 y el 2015 en la región mazahua del estado de México, en el marco del programa de Maestría en Gestión Sustentable del Agua en el Colegio de San Luis y con el apoyo financiero del Consejo Nacional de Ciencia y Tecnología de México. 
amenaza la persistencia de un modo de vida particular que, en condiciones de desigualdad socioeconómica, hace parte de la trama de relaciones socionaturales que constituyen el paisaje hídrico y en donde el agua, la tierra y el maíz son los elementos que fundamentan su ontología.

En este texto, hablar de ontología supone la existencia de múltiples realidades en relación con el agua; diversas formas de experimentar el mundo natural mediante las cuales las sociedades, situadas histórica y geográficamente, configuran propiedades que identifican al agua. Esta perspectiva es pertinente porque nos permite reconocer otras "creencias" de un grupo "tradicional" con respecto al agua u otras formas de explicarla y darle sentido. Pero las ontologías del agua no tienen que ver únicamente con qué saberes o conocimientos están más cercanos a la realidad -lo que configuraría un conflicto epistemológico-, sino con el carácter mismo de lo que existe y puede ser conocido. Así, plantean un conflicto ontológico (Blaser 2009, 2014) que interroga los fundamentos mismos de la economía política y abre nuevas formas y “puentes metafóricos” para pensar el trabajo, la producción y las subjetividades en torno al agua de maneras más genuinas y menos instrumentales (Ruiz-Serna y Del Cairo 2016).

Por otro lado, al entender el paisaje hídrico como producto y a la vez productor de una trama de interrelaciones agua-sociedad y otros elementos no humanos - que tienen una dimensión material, simbólica y política一, contribuyo a una discusión más amplia sobre la gestión del agua desde una perspectiva territorial que la reconoce, junto con el territorio, como actores interdependientes. De allí que, en el caso de los trasvases de agua, la consideración seria y participativa de las poblaciones de las cuencas aportadoras debe ser fundamental para la preservación del agua y de la vida comunitaria, tanto en los territorios de montaña como en los que están cuesta abajo. Es preciso buscar una reciprocidad entre las ciudades consumidoras y las localidades indígenas y rurales proveedoras del líquido, ya que estas últimas han permanecido en la marginación y la pobreza extrema sin recibir una compensación real y eficiente por los recursos que aportan al desarrollo regional.

Este estudio se sustenta en el concepto de paisaje hídrico y la perspectiva territorial de la gestión del agua. Su pertinencia radica en mostrar que, en el caso mazahua, el paisaje hídrico del territorio no solo incluye el agua en diferentes formas (ríos, laguna, lluvia, manantial, etc.), sino también las montañas y otros elementos que interactúan con ella. Es decir, con elementos no humanos del paisaje que juegan un papel significativo en las prácticas y ontologías del agua, en las que el aprovechamiento de bosques y suelos húmedos son determinantes para el sostenimiento ecosistémico así como de la vida misma, en aspectos tan importantes como el sistema alimentario. 
El análisis que se presenta a continuación es el resultado de un trabajo etnográfico realizado en los municipios de Villa Victoria, Villa de Allende, Valle de Bravo y Donato Guerra en el Edomex. Hice observaciones de campo en toda la región mazahua impactada por la infraestructura del Sistema Cutzamala, y principalmente en los municipios mencionados. Allí hice entrevistas con mujeres y hombres que se identifican como indígenas mazahuas o campesinos, y con otros actores pertenecientes a los organismos de la cuenca. En Donato Guerra llevé a cabo un trabajo etnográfico más intenso y prolongado, entre los años 2013 y 2015, en las comunidades de San Antonio y San Simón de la Laguna, acompañando su vida diaria familiar y comunitaria. Frente a la negativa a ser entrevistados de autoridades y trabajadores encargados de manejar y mantener la infraestructura del trasvase del Cutzamala, hice una revisión y análisis de distintos materiales oficiales emitidos por el gobierno federal y la Comisión Nacional del Agua con respecto a esta infraestructura.

\section{El paisaje hídrico y la gestión territorial del agua}

De manera muy general se dice que el paisaje es la forma en que el espacio es visto a través de las diferentes culturas (Wagner y Mikesell 2003). Sin embargo, aquí enfatizo que si bien el paisaje es un proceso cultural vivo, no se trata de un repertorio que cada sociedad carga consigo e impone sobre un medio físico particular. En aras de comprender el paisaje no podemos abordarlo como un objeto, debemos mirarlo como un proceso en movimiento que hace a los seres humanos y a su vez está hecho por ellos (Ingold 1993).

Asimismo, las formas del paisaje se generan por el movimiento. Estas formas, sin embargo, están disponibles en un medio material incluso mucho tiempo después de que el movimiento que les dio origen haya cesado; de hecho, "un paisaje es la apariencia más sólida en que una historia puede declararse a sí misma” (Inglis 1977, en Ingold 1993). Las estructuras sociales, las significaciones simbólicas, las actividades productivas y las relaciones de poder desempeñan un papel crucial en los procesos de configuración de los paisajes a lo largo del tiempo, es decir, el paisaje no es solo "natural”, sino social, político, heterogéneo y cualitativo.

Desde la perspectiva del habitar propuesta por Ingold (1993), el paisaje se constituye como un registro - y testimonio- duradero de las vidas y las actividades de las generaciones pasadas que lo habitaron, y que al hacerlo dejaron en 
él algo de sí mismos: “el paisaje cuenta —o más bien es- una historia” (Ingold 1993, 168). Mosaicos de parcelas cultivadas, terrenos destinados para animales, bosques preservados o degradados, cursos de agua libres o canalizados, caminos y asentamientos humanos construidos con peculiares formas por las condiciones que imponen el clima, el relieve y los cuerpos de agua son el resultado de la interacción de las sociedades con los elementos de un espacio natural determinado y también de la forma en que este afecta la organización social (Altieri y Koohafkant 2015).

Si bien el paisaje hídrico también es un espacio físico con elementos tangibles, visibles y audibles, estos pueden tener uno o varios significados simbólicos o interpretaciones subjetivas. Tales significados e interpretaciones del paisaje hídrico son representaciones del territorio que también orientan prácticas y formas de manejo, por lo cual el paisaje hídrico es una práctica del habitar, un paisaje de tareas (Ingold 1993). La representación social del agua y del territorio - como toda representación social-opera como guía potencial de las prácticas y de las decisiones territoriales, pero también como forma de apropiación de estas (Giménez 2007).

En este artículo analizo de manera particular el papel del agua en la composición de los paisajes, a partir de la idea de que el agua tiene cierto movimiento que la distingue de otros bienes naturales: el agua fluye constantemente y cambia a través de geografías físicas, pero también a través de distintos contextos culturales, sociales y simbólicos (Swyngedouw 1999, 2004). En otras palabras, muestro que comprender el paisaje desde la dinámica del agua -como un paisaje hídrico- nos permite mirar un paisaje liminal, fugaz, dinámico y transgresivo (Karpouzoglou y Vij 2017).

El paisaje hídrico se entiende e interviene en función de distintos contextos y diversos sujetos sociales, de allí su potencial político y contencioso (Pérez 2006). Por ello su análisis debe considerar las distintas formas en que se habita, se percibe e interviene, pues en un mismo paisaje se encuentran diversas miradas e intervenciones que pueden incluso confrontarse. Así, el concepto de paisaje hídrico permite sostener un análisis de carácter relacional entre agua y sociedad y, al mantener abierta la pregunta ontológica de qué es el agua, mostrar que los mundos del agua son también mundos de poder. En ese entendido, la perspectiva de la ciencia occidental sobre el agua, basada en gran parte en la hidrología y la ingeniería, es solo una entre otras tantas.

El paisaje del que hablo en este artículo responde a la propuesta ecopolítica de pensar el agua como un híbrido inquieto (Swyngedouw 2004). Es decir, pensar los paisajes sensibles a los procesos dinámicos a través de los cuales el agua, 
como socionaturaleza, se reelabora continuamente -incluidos sus diversos flujos y usos-para comprender los complejos desafíos hídricos interdependientes, asociados con el acceso, la distribución y las crecientes demandas del líquido por la expansión urbana.

En específico, este artículo pretende abonar a la discusión sobre los paisajes hídricos desde la antropología, mediante la exploración de las relaciones hídricas más allá del suministro tradicional de agua por tubería, destacando la importancia y las características particulares de las relaciones socionaturales con lugares de agua no tan explorados como los manantiales, la lluvia y el agua subterránea. Destaco la importancia que tienen elementos no humanos como las montañas, los suelos y los bosques en la configuración de los paisajes hídricos desde la perspectiva de la territorialidad, es decir, la imprescindible relación aguaterritorio para la apropiación de los paisajes a partir de la gestión de los bosques y de los recursos simbólicos. Estos últimos, más allá de ser alegorías de una cultura ancestral indígena, dan cuenta de una ontología política capaz de reinterpretar, de organizar comunidades y de enfrentarse a otros habitares del paisaje hídrico implícitos en su constante intervención o transformación histórica.

Considero que la perspectiva territorial permite el análisis de asuntos que pueden pasar desapercibidos con la búsqueda únicamente del agua que se almacena y se conduce a través de la infraestructura y no del agua presente en diversas formas y aprovechada de diferentes maneras a lo largo y ancho de un territorio. Así, mi análisis parte del reconocimiento de los espacios de agua ocultos, no se limita a aquellos cuerpos, corrientes o reservas de agua que son fácilmente perceptibles por los sentidos, sino que se interesa por formas sofisticadas en las que el agua se hace presente y que solo a partir de observaciones finas y minuciosas se pueden aprovechar. Así, para el caso específico de esta investigación, el reconocimiento y la valorización del agua que cae en forma de lluvia y después se infiltra por los suelos, humedeciéndolos por largas o cortas temporadas, es una muestra de la lectura sofisticada de los campesinos mazahuas sobre el paisaje hídrico.

Por otro lado, la relevancia teórica de abordar el agua en relación con el territorio es urgente y necesaria porque moviliza diversos componentes culturales y políticos pertinentes para analizar los paisajes hídricos en conflicto. Uno de los componentes de este análisis es la importancia de la gestión comunitaria, entendida como una forma de construir territorios. En ese sentido, las formas de apropiación de espacios y recursos locales por medio de prácticas campesinas como la pesca, la recolección y la agricultura resultan también en diversas formas de gestionar agua y tierra, en pocas palabras: formas de construir territorios. 
Así, el papel del territorio en los temas de agua cobra una dimensión política fuerte, pues situar distintas prácticas y conocimientos locales en torno a un espacio apropiado (entendidos como productos históricos y comunitarios) involucra un ejercicio de gobierno y de derecho por localización que trasciende las decisiones diarias de los campesinos acerca de su territorio.

\section{El territorio mazahua y su contexto social}

El paisaje hídrico del que hablaré aquí corresponde a un territorio boscoso en el centro de México que comprende pequeños valles intermontanos, con un gradiente altitudinal que va de los 2.700 a los $2.619 \mathrm{~m} \mathrm{~s}$. n. m. Configura un relieve abrupto con barrancas y laderas, mientras que algunas elevaciones forman planicies o mesetas. Es una región parteaguas de la subcuenca del Balsas conocida como Cutzamala.

Las comunidades indígenas jñatjo, también conocidas como "mazahuas", han habitado históricamente esta región noroccidental y centro-occidental del Edomex desde el año 900 d. C. Aunque, como detallaré más adelante, a lo largo de las distintas etapas históricas y por diversos actores económica y políticamente poderosos, se vieron desplazadas poco a poco de las planicies centrales a las sierras que hoy habitan.

Aquí me refiero sobre todo a las poblaciones mazahuas que habitan la parte alta de la cuenca del Cutzamala al sureste del Edomex. Dichas comunidades se encuentran bordeadas por una serranía boscosa, de donde, a partir de la retención y los escurrimientos de agua, brotan diversos manantiales, resbalan riachuelos y se alimentan cuerpos de agua más grandes, como lagunas, en las partes más bajas. El complejo relieve de este territorio hace que la temperatura sea variable y genere diversos microclimas en un área reducida, como en el caso de las zonas ribereñas que tienen un clima más cálido, a diferencia de las áreas más frías contiguas a los montes. El paisaje de este territorio es una compleja mezcla de clima, suelos y vegetación, cuya combinación se expresa en una riqueza biológica significativa para la región centro del territorio mexicano.

Dicho territorio es un modelado paisajístico donde pueden verse trazos hechos desde la conciencia práctica de los sujetos; los huertos familiares, las milpas, el bosque al pie de los cerros, los cuerpos de agua y sus riberas son espacios adaptados y trabajados por las familias mazahua. Estas áreas son adecuadas continuamente para la siembra y el almacenamiento de maíz, frijol y calabaza 


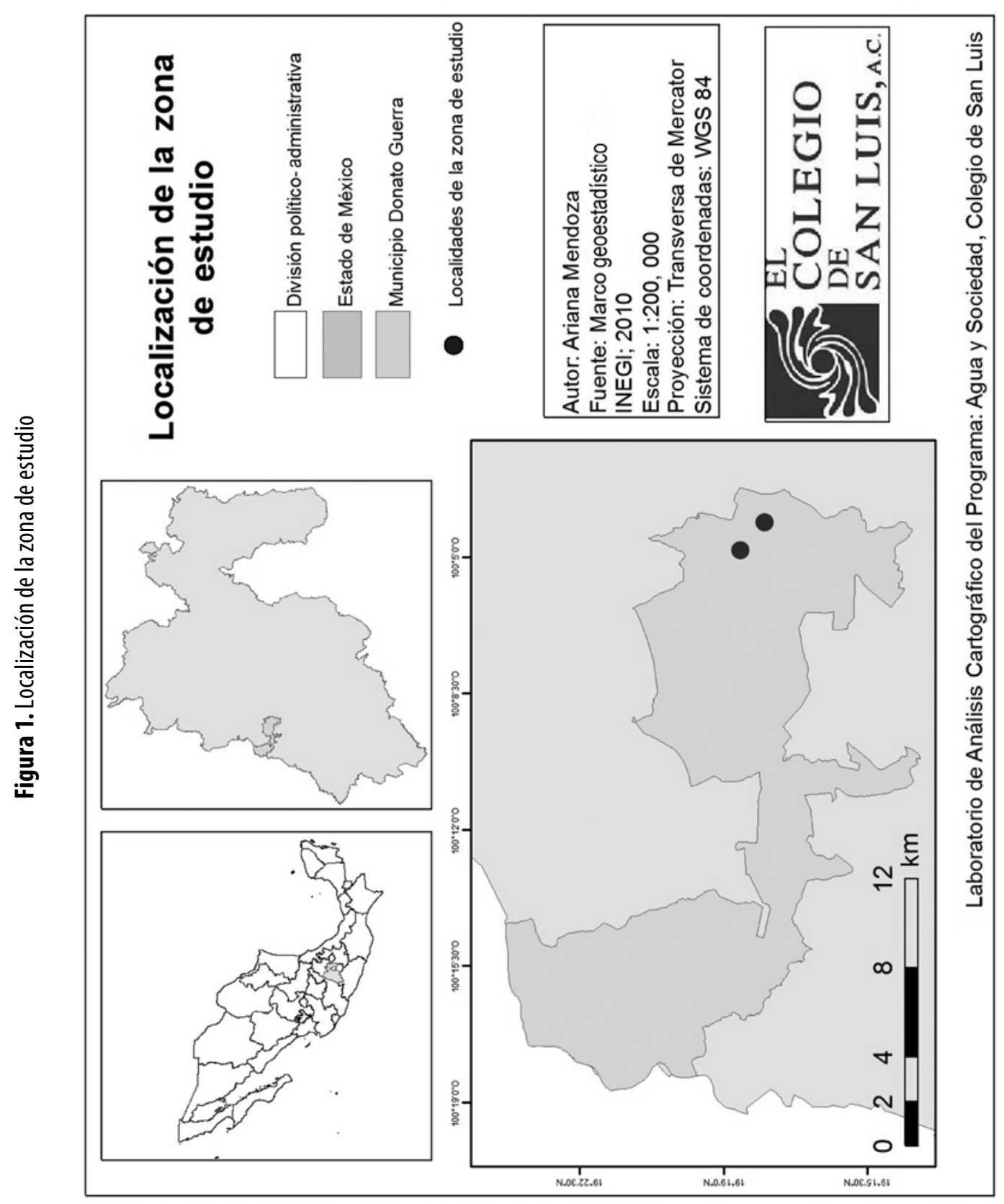

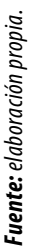


destinados al autoconsumo de las familias, así como a la recolección de algunos otros alimentos obtenidos en los cuerpos de agua o en el bosque, tales como hongos (en época de lluvias), quelites (hierbas silvestres comestibles), especies lacustres y algunas frutas.

Para entender el paisaje hídrico en este territorio es preciso contextualizar históricamente a las comunidades mazahuas que lo habitan. Estas, si bien se encuentran en un área de anidamiento en la sierra mazahua, mantienen una movilidad constante hacia otras regiones del país, principalmente por prácticas de comercio informal y por las interacciones con el Estado, a través de programas sociales asistencialistas, mediante la asignación de recursos económicos por familia, o de alimentos, como despensas y comedores comunitarios (Vizcarra 2006).

Las comunidades mazahuas de esta zona tienen una larga tradición migratoria. Durante la Colonia y los primeros años del México independiente, bajaban temporalmente de las montañas a los valles centrales a trabajar como jornaleros en las minas o haciendas de raíz de zacatón (Muhlenbergia macroura) para luego regresar a sus comunidades. Aunque durante el México posrevolucionario sus tierras fueron legalizadas bajo el régimen comunal que se mantiene hasta ahora, se enfrentan al problema de la incertidumbre climática y la complejidad de cultivar en relieves tan agrestes, de ahí que los movimientos migratorios se hayan mantenido y poco a poco hayan ido aumentando.

Durante la llamada “Revolución Verde” (1960-1970), la región mazahua del noroeste del Edomex fue objeto de políticas agrícolas que propiciaron el cultivo de maíz a gran escala, y fue así como hombres de las comunidades de la sierra, principalmente, se incorporaron como mano de obra en los grandes cultivos de maíz de San Felipe del Progreso, Toluca, Lerma y Atlacomulco, todos municipios al noroeste del Edomex. Sin embargo, desde principios de la década de 1980, comenzó una creciente migración de mujeres, hombres y hasta familias enteras a las grandes ciudades cercanas como Toluca (capital del Edomex) y Ciudad de México. Esto fue resultado de la débil presencia estatal, sobre todo en la provisión de servicios de salud y educación, y por la falta de alimentos que padecen las familias frente a fenómenos climáticos inesperados como sequías prolongadas.

Como resultado de las condiciones geofísicas de su territorio, las comunidades mazahuas de la sierra son vulnerables a las hambrunas cuando el clima y las condiciones meteorológicas no las favorecen. Frente a este panorama han tenido que leer muy bien los ciclos, a veces inciertos, de su entorno, así como aprender a localizar lugares y especies de manera detallada. La alimentación de estas comunidades depende en gran medida del maíz que pueden cosechar mediante la agricultura de temporal y de lo que se puede aprovechar mediante 


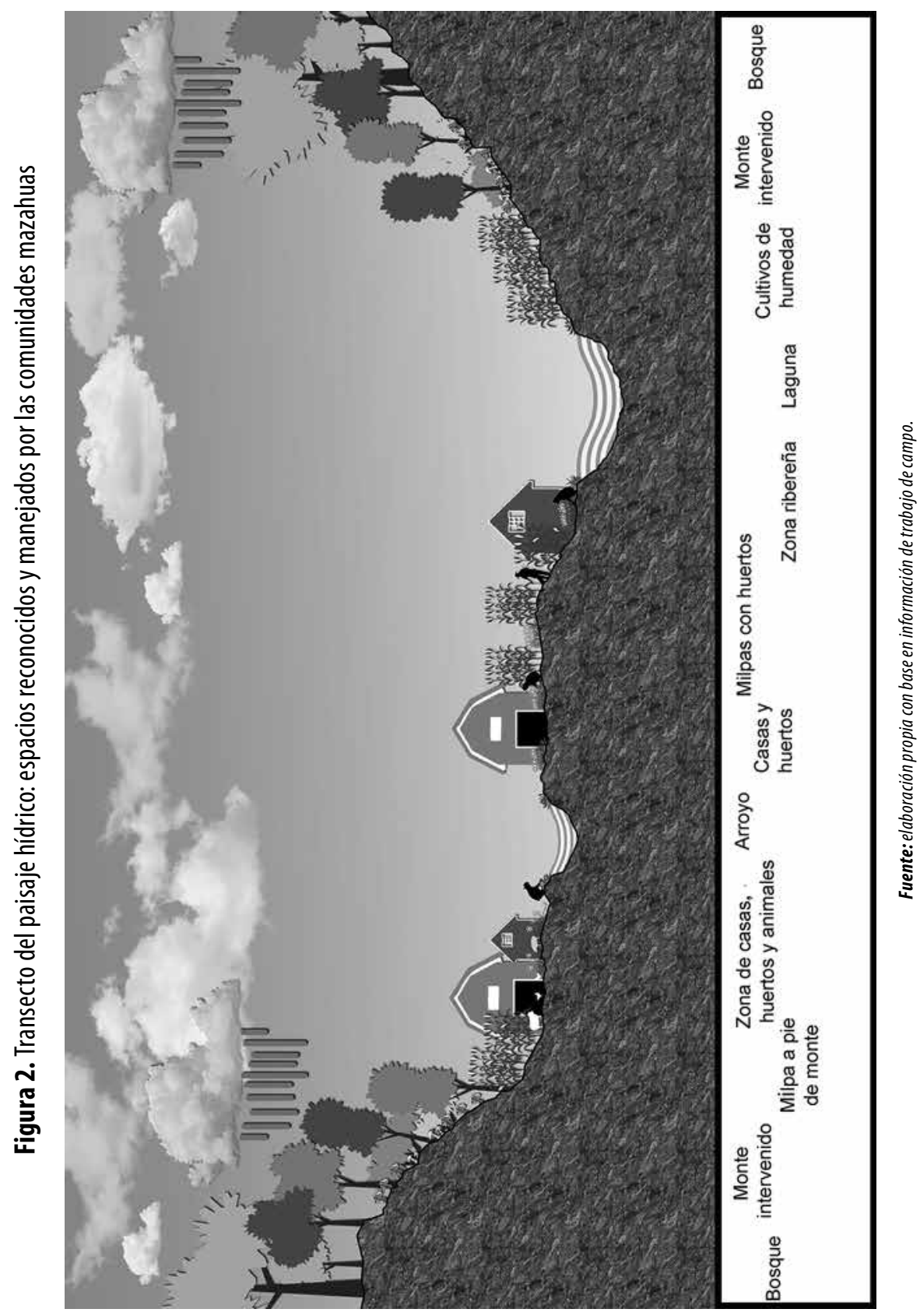


la recolección y la pesca. Complementan su dieta con ingresos monetarios obtenidos del comercio de algunas artesanías en las grandes ciudades.

La figura 2 muestra el mosaico de espacios y transiciones que son el escenario en el que las familias mazahuas habitan y, a partir de estrategias de uso múltiple, manejan el paisaje hídrico y satisfacen algunas necesidades alimenticias, de acuerdo con sus particulares condiciones espacio-temporales y su propio contexto sociocultural. Dentro de las relaciones sociedad-naturaleza que refleja el paisaje hídrico, una de las más representativas es la alimentación. Como práctica cultural, la dieta mazahua se basa en productos provenientes de su paisaje, como las tortillas de maíz (xëdyi) que acompañan todo el año los distintos platillos que hay de un periodo a otro, según la época del año.

El maíz -y su sistema de cultivo milpa- ordena gran parte del territorio, pues tanto las partes planas como los pies de montaña y los espacios lacustres, como la laguna, son intervenidos y adaptados para su siembra (Vizcarra 2001, 2002). De acuerdo con Bonfil (1987), se puede sugerir que "hay una adecuación recíproca del maíz al hombre y del hombre al maíz” (35), adecuación que no solo es territorial sino también temporal, pues el sistema de cultivo de maíz, la milpa, se encuentra íntimamente relacionado con los periodos de lluvia. La adecuación del terreno para el maíz, la forma de aprovechar el agua y la humedad de los suelos, así como la distribución y organización de los espacios han ido esculpiendo el paisaje que habitan, de forma que a lo largo de los valles altos y de las montañas se ven casas dispersas con sus respectivas parcelas de maíz.

\section{Ontologías del agua: la perspectiva mítica de los mazahuas}

Por ontologías del agua entiendo, junto con Yates et al. (2017), la posibilidad y la política de una multiplicidad de mundos relacionados con el agua, destacando múltiples realidades del agua y formas de estar con esta, no solo diferentes percepciones o sistemas de conocimiento ligados a su existencia material. Al indagar por las implicaciones del giro ontológico para los debates sobre la gestión del agua, contribuyo a explorar su agencia y cómo el agua da forma a nuestros mundos sociales a través de múltiples compromisos materiales y ensamblajes, en los que las ontologías también son un lugar de competencia política. Al enfocarme en los espacios políticos donde se encuentran las ontologías del agua, no me centro en una ontología individual sino en las implicaciones prácticas y 
políticas de habilitar o silenciar mundos diversos, que ponen en tela de juicio los supuestos ontológicos que sustentan las formas dominantes de gestión del agua (Yates et al. 2017).

Bajo ese presupuesto, para entender la ontología del agua desde las comunidades mazahuas es muy importante considerar el vínculo con el territorio ancestral con el cual guardan una fuerte relación simbólico-afectiva, pues allí se encuentran sus santos protectores y antepasados. Como lo plantea Oehmichen (2002): "el territorio continúa siendo [para los mazahuas] una de las formas de objetivación y anclaje simbólico de su identidad” (77). En esta relación simbólico-efectiva, los montes, los ríos, la laguna y los ojos de agua se integran como geosímbolos y referentes continuos de la tradición oral, de los mitos y la ritualidad de las comunidades. Su territorio se constituye en un territorio cultural, apropiado mediante el trabajo con la tierra y los significantes que se le han atribuido, lo cual ha resultado en la configuración de un paisaje hídrico en donde el agua y las montañas son parte fundamental en tanto encierran profundos significados y conocimientos para este pueblo.

Los cerros y los lugares de agua ayudan a identificar los territorios de cada una de las comunidades y, en ese sentido, el conjunto agua-cerro, en el contexto de la sierra mazahua, así como en muchos otros pueblos indígenas y campesinos del centro de México, hace referencia a una comunidad organizada asentada en un territorio (Ávila 2007). Si se piensa en relación con su principal actividad productiva - la agricultura de temporal-, el conjunto agua-cerro está vinculado no solo con la tierra sino con uno de los elementos más importantes de la naturaleza para los indígenas mazahuas: la lluvia.

Como entidad indispensable para la reproducción de la vida, al agua se le atribuye un fuerte sentido de fertilidad. Por ello existe un uso simbólico de diversas figuras femeninas asociadas al agua. De allí que, como alegoría del vientre materno, para las comunidades mazahuas el interior de los cerros sea el lugar donde se concibe y encuentra almacenada el agua que corre por su territorio y también la que cae del cielo.

En la comunidad de San Antonio de la Laguna, por ejemplo, los abuelos cuentan que dentro del cerro que llaman Tsi'swarame vive una sirena: la cuidadora y dadora de agua, de la vida. Por eso, en la parte más alta del cerro han colocado una cruz y un altar, a los que llevan ofrendas de comida y flores durante las celebraciones de Semana Santa de cada año, para que no se enoje con ellos y siga dándoles agua; se cuenta que, si un día se enoja, meneará su cola y sacará toda el agua del cerro e inundará la comunidad. Estas ofrendas van acompañadas de faenas comunitarias organizadas por los comités de agua comunitarios que, en el 
caso de San Antonio de la Laguna, se hacen para limpiar y dar mantenimiento a la infraestructura hidráulica de las comunidades, como canales, depósitos, pozos y bombas de agua.

Dado que la mayoría de las familias mazahuas depende de la temporada de lluvias para producir sus alimentos, son más vulnerables a las variaciones del clima; sequías prolongadas, heladas tempranas e inundaciones súbitas pueden arruinar su única cosecha del año. Por esta razón, su relación con la naturaleza es ambivalente: mantienen una actitud de profundo respeto y de veneración, pero también de temor por la incertidumbre acerca de cómo esta pueda comportarse (Tirel 2013).

Con respecto a la lluvia, existe un simbolismo asociado a las serpientes que son referidas por los mazahuas como "cuidadoras", "anunciadoras” o "hijas" de la lluvia. Es frecuente encontrarlas en los alrededores de las fuentes de agua y en las zonas más húmedas como el monte; se dice que están allí para custodiar el agua. La presencia de estos animales aumenta cuando la temporada de lluvias está por comenzar y para los mazahuas esto es un indicio de las lluvias: "salen a montones, pa' anunciar que ya viene el agua, bajan de allá del cerro”. Existen narraciones que cuentan que anteriormente, en época de lluvias, se veían dos víboras gigantes que bajaban del cerro. Eran tan grandes que la gente se espantaba y para ahuyentarlas se solía quemar chile guajillo en las casas.

El fuerte vínculo entre la serpiente y la lluvia hace posible la animación de esta última a través de la primera, a la que le confieren poderes sobrenaturales. En la memoria colectiva existe la idea de que, si se mata o maltrata una víbora cerca de alguna fuente de agua, esta puede secarse o mandar alguna enfermedad, susto o desconsuelo:

Dicen que sí pasa, que el hijo de don Pedro iba una vez ahí subiendo, hace unos años, que iba para el camino rumbo de San Sebastián y que se le aparece una víbora grandota ahí por donde está el charcote donde el panteón, y que de lejos la miró, que estaba grandota, dice. Y que ya cuando iba acercándose él, que la víbora como que ya no la miraba, no tan grande pues, y que sentía miedo de que se iba a desaparecer, pero que ya la vio, y que como lo enmuinó [lo hizo enojar], que por que salió que era más chica, que le dijo: "chingadera” y que se va. Ya luego, dice, que de la enmuinada se puso remal, que le dolía la panza toda la noche y se puso bien enfermo, que no saben si por hacer coraje o el susto o qué, pero que bien malo andaba el pobre por la culpa de esa viborita que le mandó el mal. (Entrevista, agosto del 2014)

De manera que para los mazahuas las conductas de los animales no son aleatorias o desordenadas, sino que constituyen relaciones que han sido 
codificadas, significadas y simbolizadas por la cultura y así pasan a formar parte de ceremonias y rituales. Por otro lado, la comprensión del paisaje y sus ciclos contempla una red de relaciones dinámicas en la que actores - humanos y no humanos-, por el hecho de compartir un territorio, hacen cosas que tienen implicaciones colaterales e imprevisibles, pero que pueden ser mediadas por el intercambio de orden anímico y energético reflejado en las ofrendas a sus santos - espacios naturales sagrados y los rituales católicos-, que guardan una estrecha relación con los ciclos naturales y de trabajo agrícola.

Uno de los símbolos más importantes de la región, asociado al paisaje hídrico, es el de la Sirena, un personaje mitológico con fuerte presencia en el imaginario colectivo de la región mazahua. La Sirena, híbrido de una mujer con cola de pescado o serpiente, ha sido registrado por Albores (1995) y otros autores (Maruri 2003; Romero-Contreras 2005; Ruiz y Delgado 2013) en el territorio del Valle de Toluca e Ixtlahuaca ${ }^{2}$. En estos análisis se le ha considerado la señora de los humedales que da vida al ecosistema, y en general a la biodiversidad, por lo que se puede entender como una representación tanto de la fertilidad como de la regulación y los límites a la explotación de los bienes lacustres disponibles. De ahí que en la mayoría de las narraciones en las que se hace presente, la Sirena tome la vida de los humanos que no respetan sus límites.

Para la comunidad de San Simón de la Laguna, el santo patrono y el espíritu de la laguna que se forma en la parte baja de su territorio son los encargados de darle paz y prosperidad al pueblo, pues se habla de una fuerza energética que tiene la laguna que la protege a sí misma y a la comunidad en general: personas, tierra, bosques y agua. Dicha energía es nombrada en lengua mazahua como Menyeje, el espíritu o dueño del agua, que se hace presente a los ojos de las personas de formas distintas, las más frecuentes son como víbora, mujer y pez. He aquí su vinculación con la alegoría de la Sirena.

En la tradición oral, la laguna forma parte de la trama de relatos que hablan de los seres que la habitan y de ciertos eventos que tuvieron lugar allí. Mediante las narraciones se establecen las explicaciones de los valores atribuidos a la laguna y las normativas con respecto a su aprovechamiento: “el Menyeje ahoga a los que vacilan con la laguna [...] con esta lagunita no se puede jugar, esa lagunita se defiende” (entrevista, octubre del 2015). matlazinca antes señalada, no solo por la evidencia material de especies como las ranas, los charales y acociles que todavía se siguen consumiendo o se consumían hasta hace unos años. Los referentes simbólicos del paisaje lacustre también dan muestra de que la cultura lacustre desarrollada en el altiplano mexicano fue de una gran riqueza y valor para el mantenimiento de las sociedades prehispánicas, incluso después de la llegada de los conquistadores. 
El mito del Menyeje o “el espíritu de la laguna” sintetiza dramáticamente los valores y prácticas generadas por la cultura lacustre, al tiempo que encarna la dimensión simbólica de la destrucción y pérdida de este modo de vida. En ese sentido, estos símbolos lacustres pueden considerarse como la última batalla de las comunidades mazahuas para mantener viva la memoria colectiva y sobrellevar la modernidad en ciernes (Trejo y Arriaga 2009).

Por otro lado, los mitos no solo dejan ver la importancia de la laguna en el sistema de conocimiento y la ontología del agua indígena, sino que van actualizando el sentido epistemológico de estas narrativas. Para el caso del mito del Menyeje (la Sirena), hay una relación con la lectura del ciclo anual de la laguna y de su temporada de veda, es decir, explica alegóricamente por qué en determinada época es más difícil realizar la pesca:

[...] cada temporada de lluvia el espíritu del agua se tiene que alimentar, hace que los peces salgan hasta la superficie, entonces algunas personas de San Simón y de otras comunidades se acercan a pescar, pero como el espíritu de este lago cuida que no se acerquen porque le toca comer, ahoga a los pescadores. (Entrevista, mayo del 2012)

Así, la ontología del agua de los indígenas mazahuas se articula al devenir de las actividades productivas a partir de la observación minuciosa de su paisaje, los ciclos, cambios y elementos que observan en él. De tal forma, las representaciones sociales del paisaje hídrico tienen una gran practicidad, dada su dimensión epistémica, pues las representaciones del agua guían la manera en la que los mazahuas lo intervienen y manejan en función del reconocimiento de sus elementos y ciclos a través de los mitos.

En este paisaje hídrico se encuentra una serie de adecuaciones que dan cuenta de un manejo territorial del agua por parte de unos actores, en el que la lluvia y otras presencias del agua menos patentes, como los mismos bosques y la humedad de los suelos, son imprescindibles. Pero en este mismo paisaje también concurren una serie de intervenciones que se caracterizan por tener un impacto de mayor escala en el territorio y que privilegian otros espacios y manifestaciones del agua, es decir, el agua que puede ser represada, "potabilizada", entubada y trasvasada. Tal es el caso de la infraestructura de captación, represamiento y trasvase que se encuentra en esta misma región y que tiene como destino la Zona Metropolitana del Valle de México. 


\section{El agua desde la racionalidad moderna capitalista}

El crecimiento acelerado de la Ciudad de México (CDMX) y su área metropolitana, iniciado a principios del siglo XX -y que se mantiene hasta la fecha-, ha seguido una lógica centralista y voraz que avanza sobre las zonas rurales que la rodean, acaparando tierras, bosques y aguas, y desarticulando comunidades. En esa tendencia, el Edomex ha servido a la CDMX como un territorio proveedor de los diferentes recursos naturales según sus necesidades. A finales de la década de 1930, la CDMX requería más energía eléctrica para abastecer a su creciente población y desarrollo industrial, por lo que se construyó una gran infraestructura hidroeléctrica al noroeste del Edomex. Posteriormente, necesitó más agua y esa misma infraestructura fue utilizada y ampliada para la dotación del líquido, de manera que "los ríos y los valles altos del estado de México que eran espacios de vida para los mazahuas se transformaron en canales y reservorios de agua para la ciudad" (Tirel 2013, 103).

Para abastecer de agua potable a la industria y a la población en aumento en la capital del país en la década de 1940, se comenzaron a explorar nuevas opciones de abastecimiento más allá de la cuenca del Valle de México, pues ya se habían sobreexplotado sus mantos acuíferos y contaminado o entubado sus fuentes superficiales. Así fue como se decidió construir el primer trasvase de cuenca en la historia de México, mediante el sistema Lerma en 1951. Sin embargo, la demanda de agua seguía incrementando y, además de aprovechar el agua de las lagunas de la cuenca del Lerma, también se perforaron más de ochocientos pozos profundos, lo que provocó el desecamiento de las lagunas y el abatimiento de los niveles de los acuíferos de esa zona (Legorreta 2006).

Con el agotamiento de los recursos hídricos de la cuenca del Lerma se decidió construir un sistema de trasvase de una cuenca un poco más alejada, aprovechando la infraestructura ya establecida en el Sistema Hidroeléctrico Miguel Alemán. En la década de 1980 se inició la construcción del Sistema Cutzamala en varias etapas, para captar $19 \mathrm{~m}^{3} / \mathrm{s}$ en total, aprovechando las corrientes de los ríos Malacatepec, Valle de Bravo e Ixtapan del Oro en el Edomex, así como las de los ríos Tuxpan y Zitácuaro en Michoacán - todos pertenecientes a la subcuenca del Cutzamala, afluente del río Balsas-. Para ello se construyó un conjunto de siete presas, las dos más grandes, Valle de Bravo y Villa Victoria, con una capacidad de almacenamiento de 400 y 210 millones de metros cúbicos respectivamente (Conagua 1997, 2005). 
La primera etapa del Sistema Cutzamala fue inaugurada en 1982 y consistió en captar $4 \mathrm{~m}^{3} / \mathrm{seg}$ de la presa Villa Victoria y conducirla, por un acueducto de 2,5 m de diámetro, a través de $77 \mathrm{~km}$ atravesando las sierras de Las Cruces al poniente de la CDMX. La segunda etapa entró en operación en 1985 y aportó 6 m³/seg de agua de la presa de Valle de Bravo, potabilizada antes en la planta de Los Berros, para luego recorrer $29 \mathrm{~km}$ de canales que vencen una altura de $1.100 \mathrm{~m}$ ayudados por tres plantas de bombeo. En 1993 comenzó a funcionar la tercera etapa que represa y trasvasa $1 \mathrm{~m}^{3} / \mathrm{seg}$ de agua de la presa Chilisedo y $8 \mathrm{~m}^{3} / \mathrm{seg}$ de la presa Colorines, que a su vez recibe aguas de las presas Tuxpan, El Bosque, Ixtapan del Oro y Tilostoc. En total, en los primeros años del siglo XXI, cuando ya estaban funcionando estas tres etapas, el Sistema Cutzamala aportaba un promedio de 20 $\mathrm{m}^{3} /$ seg a la CDMX y su zona conurbada (Conagua 1997, 2005).

Dicha infraestructura ha modificado drásticamente el territorio mazahua. Sin duda, el trasvase manifiesta una forma particular de habitar el paisaje, en la que el control del agua, y no el entendimiento y la adecuación a sus ciclos y dinámicas, es el eje impulsor. Los más de $300 \mathrm{~km}$ de tuberías y canales que atraviesan las sierras para llevar el agua a la ciudad hacen parte del ensamblaje de ontologías del agua que dan sentido y configuran el paisaje hídrico, sin embargo, en este caso representan también, para algunas comunidades mazahuas, un impacto negativo en sus vidas. Para los gobiernos federales y estatales, en cambio, este proyecto, la tecnología de punta y su sistema de construcción representan un éxito de la ingeniería mexicana, motivo de orgullo e insignia del progreso, sobre todo porque el Cutzamala ha transformado de tal forma el paisaje hídrico de la región, que ha mostrado que el agua puede captarse y conducirse según las necesidades de ciertos actores sociales. Es un entendimiento de que el paisaje hídrico siempre se puede controlar.

Con lo anterior no quiero decir que los mazahuas no hacen modificaciones a su paisaje hídrico. Claramente lo intervienen y aprovechan para satisfacer sus necesidades alimentarias, principalmente; pero son dos maneras de intervención del paisaje hídrico que se diferencian en escala e intensidad, y están motivadas por valorizaciones distintas del agua. El Sistema Cutzamala impacta de manera más intensa y a una mayor escala los ciclos ecosistémicos, da prioridad al agua susceptible de ser represada sin importar cómo puede alterar un ciclo hidrosocial más extenso y las relaciones agua-sociedad de la cuenca "donante”. Además, ha provocado impactos socioambientales severos, como deforestación, contaminación de ríos y mantos acuíferos, pérdida de biodiversidad, desecación de manantiales, generación de desechos industriales y residuos sólidos, entre otros, en sus casi 36 años de operación. 
Como bien lo ha señalado Tirel (2013), la región del Cutzamala tiene "paisajes engañosos”. Los altos y verdes bosques, los numerosos cuerpos de agua, así como los múltiples embalses que se observan por toda la región, ofrecen la imagen de un paisaje con abundante agua. Pero detrás de esos verdes paisajes hay una escasez que ha sido invisibilizada y que se comenzó a complejizar en los últimos años con la presencia y operación del Sistema Cutzamala. Tal como lo señala una mujer mazahua, habitante de una de las comunidades aledañas a dicha infraestructura, en un testimonio recuperado por Tirel (2013): “de nada sirve tener el agua cerca, de nada sirve ver las presas muy cerca de sus casas si para tenerla hay que acarrearlas en cubetas y caminar 4 kilómetros con el chamaco en la espalda y el bulto de ropa al lado” (115).

Antes del Cutzamala, las comunidades mazahuas carecían del acceso a agua potable en sus casas y las familias estaban acostumbradas a invertir gran cantidad de tiempo al día para acarrear agua desde las fuentes más cercanas. Con la llegada del Cutzamala, las personas comenzaron a cuestionarse esa forma de vida y a preguntarse por qué el Gobierno les garantiza el agua a unos, mandándola desde muy lejos hasta la llave de sus casas, mientras que a otras personas las deja abandonadas a su suerte sin preocuparse por que puedan acceder a este líquido. Desde entonces, y luego de una serie de afectaciones por inundaciones en terrenos contiguos a la presa Villa Victoria, en el 2004 comenzó una movilización social contra el Sistema Cutzamala que evidenció los impactos de la obra sobre las comunidades vecinas y denunció el rezago en infraestructura para los servicios hidráulicos básicos y de energía eléctrica (Gómez-Fuentes 2009, 2014).

Sin embargo, lo que se reclamaba no era solo la falta de infraestructura para dotar de agua a las comunidades mazahuas. Para la realización del Cutzamala se expropiaron tierras, y otras fueron invadidas sin indemnización o se pagaron a precios muy bajos. Con la construcción de algunas represas, como la de Valle de Bravo, se desplazó a familias enteras y desaparecieron las mejores tierras para la agricultura. La construcción de canales y la instalación de los grandes tubos que transportan el agua han atravesado grandes distancias y separado el territorio de estas comunidades. La instalación de líneas eléctricas de alto voltaje, así como la construcción de las bombas para impulsar el agua y las plantas potabilizadoras, no solo han fragmentado el territorio, sino que también han ocasionado problemas ambientales entre los que se destaca la deforestación y erosión de suelos, además de la cesión del control y vigilancia del territorio a actores externos a las comunidades.

Al contener una infraestructura hidráulica administrada directamente por la Comisión Nacional del Agua (Conagua), el territorio que habitan las 
comunidades mazahuas ha sido objeto de distintos programas federales y estatales de manejo y conservación forestal, lo que trajo consigo una serie de afectaciones en el tejido social y en el vínculo de este con los elementos no humanos de su paisaje, específicamente con el bosque que los circunda. Gran parte de la sierra mexiquense que contribuye a abastecer de agua al Cutzamala hace parte del Área Natural Protegida de la Reserva de la Biosfera de la Mariposa Monarca y del Área de Protección de Recursos Naturales "Zona Protectora Forestal los Terrenos Constitutivos de la Cuencas de los Ríos Valle de Bravo, Malacatepec, Tilostoc y Temascaltepec”. Dichos mecanismos jurídicos y su consecuente parcelación del territorio han construido una frontera simbólica y material con las comunidades mazahuas, que de manera incremental se ven más desvinculadas del monte, sobre todo por la prohibición, cada vez más contundente, del aprovechamiento de sus especies por parte de las familias locales.

Las instituciones receptoras de un préstamo del Banco Mundial, como el programa de Restauración Forestal en Cuencas Hidrográficas Prioritarias "Cutzamala-La Marquesa”, deben realizar "consultas previas, libres e informadas” con las comunidades indígenas a fin de lograr un amplio apoyo comunitario y facilitar la participación informada de los pueblos en el diseño e implementación de los planes, o para preparar acciones de mitigación o compensación (Ávila 2014). En la realidad ocurren distintos procesos que impiden la articulación de las comunidades y el beneficio de estas en conjunto. Algunos de los problemas son: la falta de derechos agrarios que impide a grupos de la comunidad (principalmente mujeres y jóvenes) formar parte del proceso de toma de decisiones sobre los bienes de uso común; la baja participación en las asambleas, derivada del desinterés por las actividades primarias y de la desconfianza hacia las autoridades agrarias; y la concentración de la información y la toma de decisiones en los asesores técnicos forestales y la mesa directiva de los núcleos agrarios. El punto más crítico es que las comunidades y sus formas de organización local han sido subvaloradas, desgastadas y desarticuladas por la injerencia de estos programas que han contribuido al debilitamiento de las instituciones locales encargadas de manejar los recursos de uso común como los bosques. La concentración de la información y la toma de decisiones entre los asesores técnicos y los líderes políticos de los pueblos ocasiona conflictos en las comunidades y entre comunidades vecinas por la falta de transparencia. En concreto, dichos programas muestran que, como consecuencia del Sistema Cutzamala, el territorio mazahua ya no es controlado, o lo es mínimamente, por los pueblos.

Lo más alarmante es lo que se comenta continuamente en la región: “en un tiempo ya ni caminando por una hora, dos horas vamos a poder conseguir 
agua, ni eso nos va a quedar” (entrevista, agosto del 2015). Aquellas fuentes naturales de agua adonde las personas acudían para llevar agua a sus casas están secándose. Con ello también han disminuido la flora y fauna que los mazahuas aprovechan para su alimentación y que forman parte importante de su ontología del agua. Al principio esta situación se había documentado en las partes más bajas de la subcuenca del Cutzamala, pero para las comunidades de las partes más altas este no había sido un fenómeno tan evidente sino hasta hace unos tres años, cuando comenzaron a notar una disminución de algunos cuerpos de agua y la desaparición de manantiales. Los pobladores cuentan que estos tal vez desaparecieron a causa de las excavaciones y explosiones con dinamita en cerros y lomeríos que hicieron para atravesar las tuberías y los canales, alterando así los flujos de agua superficial y subterránea.

También hubo una importante disminución de áreas de bosques, debido a que se tuvieron que talar montes para delinear los caminos de acceso a la infraestructura, despejar terrenos para las tuberías, canales y torres de oscilación. Esta situación ha afectado el ciclo hidrosocial y por ello los pobladores relacionan el uso de la dinamita con la progresiva desecación de ciertos cuerpos de agua. En algunos municipios se habla de la pérdida de flora y fauna lacustre ocasionada por la contaminación, a raíz del vertimiento de desechos tóxicos de las plantas potabilizadoras. Estos fuertes impactos ambientales también afectan la vida cotidiana de las familias mazahuas, quienes tienen que acudir a lugares cada vez más alejados para abastecerse de agua, e incluso terminan desplazándose a las ciudades. La condición de estas comunidades, que ya era preocupante antes de las afectaciones del Cutzamala, se agrava continuamente, pues ni el acceso directo a ciertas fuentes de alimentos se puede garantizar hoy día.

La problemática anterior responde a un modelo de gestión de agua particular y complejo que se ha vuelto hegemónico, un modelo centralista y monopolizador que, en el caso del trasvase de agua Cutzamala, es protagonizado por el gobierno federal a través de la Conagua. Este modelo también tiene su propia respuesta a qué es el agua: una mercancía, un bien material que proporciona - a algunos - comodidad, prosperidad y que esperamos obtener, según el estatus económico, lo más clara, inodora e incolora posible (Worster 2008). Un recurso natural desagregado de otros, y por lo tanto controlable, que "con un mínimo esfuerzo de nuestra parte brota de un grifo, mientras que los medios por los que llega a nosotros son dados por sentado” (Worster 2008, 163). Esa es una de las ontologías del agua dominantes en el capitalismo.

Dicha ontología fundamenta la lógica de abastecimiento de agua de la Ciudad de México, en donde históricamente ha prevalecido la idea de que se puede 
extraer agua de cualquier lugar si hay viabilidad técnica y financiera. Además, permite mantener ciertos estilos de vida en detrimento de otros, cuando se considera moralmente correcto despojar a unos para satisfacer las demandas del centro económico y político del país, mediante obras que se consideran de utilidad pública o para el beneficio del desarrollo nacional. La misma "solución” de los trasvases no ha logrado romper con esta lógica, puesto que cuando el agua llega a la CDMX tampoco se distribuye de forma equitativa. En la CDMX y su área metropolitana, los municipios conurbados y las delegaciones del norte y oriente -que son las más vulneradas económica y socialmente-reciben la menor cantidad de agua del Sistema Cutzamala, mientras que las delegaciones con más alto poder adquisitivo son las más beneficiadas.

\section{El ensamblaje de múltiples ontologías y valorizaciones del agua}

La naturaleza - y el agua - no es una entidad objetiva, sino que se entiende de acuerdo con diversas formas culturales (Babidge 2016; Boelens 2014; Descola 2012; Linton y Buds 2014). Aunque esta idea no es nueva, es importante destacarla aquí porque permite apreciar cómo las diferentes maneras de habitar el paisaje hídrico del que he venido hablando se han formado subjetivamente y no siempre coinciden; al contrario, la mayoría de las veces compiten entre sí (Swyngedouw 1995, 1997, 2004).

En el paisaje hídrico del territorio mazahua están presentes relaciones de poder materializadas en formas desiguales de acceso al agua. Una gran parte de la población mazahua no tiene agua potable disponible en sus casas aunque posea la infraestructura. La justificación discursiva de las autoridades municipales y estatales es la dispersión y la lejana ubicación geográfica de algunos hogares con respecto a las zonas centrales de las comunidades. Mientras tanto, el Sistema Cutzamala es la materialización de la ideología de desarrollo y progreso en la que la tecnología desarrollada por “el hombre” permite superar las distancias y los obstáculos geofísicos para abastecer de agua al centro financiero y político más importante del país y a una de las ciudades más grandes del mundo.

Como recorte de una realidad mucho más amplia y compleja, el paisaje narrado en este trabajo responde a un ensamblaje de ontologías y valoraciones del agua en un espacio geofísico. Las relaciones de poder desiguales también son manifiestas en la imposición de una concepción del agua sobre otra. De tal 
manera, sobre el paisaje hídrico de las comunidades mazahuas de la sierra mexiquense, gobernantes e ingenieros vislumbran un potencial hídrico en el mismo lugar donde ecólogos y biólogos contemplan un área natural protegida con especies forestales endémicas. Este es el mismo lugar que representa el hogar de los antepasados de las comunidades mazahuas y el territorio en el que han desarrollado un conjunto de conocimientos y prácticas productivas y alimentarias a partir de su relación con el entorno.

El paisaje hídrico que he descrito no solo es intervenido de maneras visibles, sino que allí es posible distinguir formas de modificación más impactantes que otras. Este paisaje desigual también muestra que existen diversas maneras de estar con el agua, múltiples realidades de esta, percepciones diferentes sobre su gestión o diversos sistemas de conocimiento ligados a su existencia material. Siguiendo la propuesta analítica de Mclean (2017), las distintas ontologías del agua que coexisten en la subcuenca del Cutzamala se producen mediante ensamblajes. Así, en lugar de desplegar un análisis de una materialidad particular, como el Sistema Cutzamala, por ejemplo, es necesario dar cuenta de cómo ciertos valores, epistemologías y prácticas en torno al agua se institucionalizan como los modelos dominantes de gestión, redundando en la marginación de otros compromisos hídricos y otras formas de estar con el agua.

Cada una de estas ontologías tiene matices y tensiones, además de no ser las únicas. Sin embargo, para fines analíticos quise caracterizar aquí dos de ellas referidas al agua, que se despliegan produciendo paisajes hídricos desiguales y que no responden necesariamente a la delimitación de una cuenca. He querido mostrar que, al coexistir, estas ontologías del agua operan como ensamblajes, iluminando los procesos a través de los cuales se combinan múltiples elementos para coproducir aguas (Mclean 2017). Si bien este enfoque es útil para descubrir múltiples dimensiones de la composición biofísica del agua en diversos paisajes, es importante destacar las ontologías del agua como un sitio de competencia política.

Ahora bien, ¿cómo se articulan estas dos ontologías y valorizaciones del agua? En principio, es claro que la perspectiva hegemónica, aquella que responde a una lógica de acumulación de capital, necesita — paradójicamente- de la ontología y epistemología indígenas. Las particularidades culturales y la racionalidad que guía las intervenciones de la población mazahua en el paisaje, así como las condiciones de desigualdad socioeconómica en la que se encuentran, han posibilitado que este reservorio de agua siga existiendo hoy. Muestra de ello es el manejo forestal que han hecho de sus montes, pese a que las formas de entender y vincularse con el agua de la población mazahua tampoco son las más amables y armónicas con el medio ambiente, pues hay casos de contaminación de fuentes de agua por usos locales, por ejemplo. 
En un mismo paisaje hídrico se ensamblan, por una parte, una ontología del agua que la valora en sus múltiples estadios y formas: en manantiales y lluvia que humedecen y se infiltran en los bosques. Por otra parte, una racionalidad del agua que la valora solo en términos de los metros cúbicos por segundo que pueda represar, desechando su vínculo con un ciclo hídrico con conexiones materiales y simbólicas que se despliegan por todo un territorio, con personas y otras formas no humanas del paisaje. Esta misma racionalidad dominante ha marginado a otros sujetos del acceso a este líquido, pues si bien los usos del agua en las comunidades mazahuas responden a una lógica territorial de su aprovechamiento, como ellos mismos lo manifiestan, también tienen derecho a tener grifos con acceso a agua en sus casas. Bajo esa idea, la garantía de acceso al agua no tiene sentido sin el pleno acceso al territorio.

En un mismo lugar convergen así procesos de percepción, apropiación e intervención del medio, acordes con diversas y disímiles formas y modos culturales. Tenemos, en este caso, miradas que se confrontan: por un lado, las que son consecuencia de la cotidianidad y de la fuerza del devenir. Por el otro, aquellas acordes con los paradigmas científicos y técnicos, alimentados por la lógica de la racionalidad instrumental y del desarrollo, forjados en un lugar ajeno, tanto del paisaje como de quienes lo habitan.

Tener en cuenta esto es fundamental para el análisis de la región montañosa que aquí se trata, en el contexto del trasvase de agua a la Ciudad de México. Sobre ese territorio boscoso y con abundancia hídrica interactúan percepciones e intervenciones distintas y en confrontación. Por una parte, existe la percepción de que este es un territorio potencialmente explotable, en tanto que el Estado y el capital lo piensan prístino e inhabitable, y por lo tanto, inaprovechable. Desde la lógica del capital, su suelo abrupto no posibilita prácticas productivas rentables, aun cuando, paradójicamente, tiene un gran valor económico para el desarrollo de las dinámicas de la ciudad y el capital.

Por otra parte, la misma región es el hogar histórico de un pueblo que valora sus bosques, tierra y agua, en la medida en que son referentes simbólicos que dan sentido a sus vidas y de los cuales dependen directamente para sobrevivir. Del aprovechamiento inmediato de ese territorio se deriva la satisfacción de sus necesidades alimenticias básicas, y también la reproducción de su cultura y su forma de ver el mundo. Símbolos, conocimientos y prácticas que se encuentran amenazadas por las consecuencias ambientales del trasvase y que incrementarían las injusticias sociales y la marginalidad en la que se hallan estas comunidades.

Es así que el análisis del paisaje hídrico mazahua debe dar cuenta de los procesos sociales, económicos y políticos que lo transforman de maneras distintas. Procesos que son tanto materiales como discursivos (Budds 2010). El 
despliegue del Sistema Cutzamala en territorio mazahua, entendido como el resultado del modelo dominante de gestión del agua y del “ambiente”, considera discursivamente a la naturaleza tan solo como un ensamblaje de componentes materiales que son objeto de manipulación humana. Es, sin duda, una representación de la naturaleza que la desvincula completamente de la sociedad, en la medida en que no considera su mutua dependencia y la entiende, más bien, como un proceso unidireccional en el que los seres humanos intervienen en la naturaleza, pero no al contrario (Budds 2010).

Frente a este panorama, se puede afirmar que el manejo del territorio no es simplemente una cuestión técnica, sino que se forma por las perspectivas de quienes lo manejan y conforme a los intereses y las demandas de los diferentes grupos sociales: comunidades indígenas y campesinas, además de grupos poderosos como gobernantes, tecnócratas y capitalistas. En ese sentido, Swyngedouw (1995, 1997, 1999, 2004) sostiene que las relaciones de poder social implicadas en el control del agua reconfiguran paisajes hídricos y, por lo tanto, mediante el examen de sus características podemos descubrir la dinámica de poder que está en juego. Estas relaciones de poder social serán aparentes tanto en los elementos materiales como en los elementos discursivos del paisaje hídrico. Por ejemplo, en la presencia de infraestructura hidráulica y en las modalidades de acceso y exclusión con respecto al agua, así como en la configuración del paisaje intervenido por los indígenas mazahuas representado en sus narrativas.

\section{Conclusiones}

Es preciso considerar el papel clave de la política hídrica en relación con el proceso de modernización nacional en un paisaje hídrico que es cambiado por la ingeniería hidráulica diseñada para transferir agua desde el territorio indígena mazahua a la metrópoli más grande del país. En este caso, se puede hablar de la coexistencia de una ontología que permea el vínculo naturaleza-cultura de forma relacional y una ontología del agua con valores asociados al proceso de acumulación de capital. Es decir, vemos la reconfiguración del paisaje milpero y lacustre hacia un paisaje caracterizado por intervenciones tecnocientíficas y áreas cada vez más amplias de deforestación, en las que las relaciones de poder son drásticamente desiguales y contradictorias.

El proceso material y discursivo por el que el Sistema Cutzamala aprovecha el control sobre el agua perfila paisajes hídricos en formas que reflejan 
los intereses y las intenciones de los agentes capitalistas, en detrimento de un paisaje hídrico basado en el manejo territorial del agua y los bosques. En conclusión, la captación y el flujo del agua intervenido con fines económicos producen paisajes hídricos geográficamente desiguales y socialmente injustos que reflejan esa acumulación.

Hay una contradicción inherente a la lógica de este ensamblaje de ontologías y valoraciones del agua que, como el analizado aquí, despliega paisajes hídricos injustos y desiguales en otros lugares del territorio nacional y mundial. Mientras la moderna cultura global de acumulación capitalista sigue expandiéndose de manera incesante, al igual que el crecimiento urbano y el número de habitantes en las áreas metropolitanas como la Ciudad de México, las ontologías indígenas y míticas del agua se desdibujan, se inferiorizan. Con ello hay una pérdida de conocimientos y prácticas locales en torno a paisajes hídricos — sin que su protagonismo en este artículo implique una romantización del pasado indígena-.

Haciendo alusión al planteamiento de Worster (2008, 175), sin herencia tradicional, sabiduría popular o tecnología local de pequeña escala; sin pozos o canales artesanales; sin manejo de zonas lacustres ni manejo local de bosques y aprovechamiento de sus especies para la alimentación local; sin organización comunitaria ni el tejido social y cultural capaces de capturar el agua necesaria para satisfacer directa o indirectamente esas crecientes metrópolis y sus demandas de consumo, ¿cuál será el futuro de nuestros territorios y paisajes hídricos?, ¿de dónde vendrá el agua para mantener nuestras tiranas ciudades del presente y el futuro?

Frente a estas cuestiones y sin pretender ser exhaustiva al respecto, mi conclusión apunta a que no podremos superar la incertidumbre frente a "la crisis por el agua” si no reconocemos las contradicciones intrínsecas y parricidas de la gestión del agua que estamos favoreciendo. Ello precisa reconocer que nuestro paisaje hídrico no es solo afectado, sino que a su vez afecta las relaciones entre los humanos así como con lo no humano, es decir, reconocer la agencia del agua y del territorio, de los bosques y montañas. También implica reconocer que en el paisaje hídrico coexisten y se despliegan múltiples ontologías que necesitan encontrar acuerdos y superar el contexto de desigualdad en el que se encuentran, pues lo ontológico es político. Incluso en sus territorios de anidamiento, los mazahuas podrían beneficiarse de las ontologías dominantes sobre el agua, mediante la ejecución de proyectos sociales participativos que respondan efectivamente a las necesidades locales y permitan a las comunidades rurales la libertad de trabajar bajo sus propios términos y guardar agua donde y cuando para ellos sea más sabiamente almacenada, según sus tradiciones. 


\section{Referencias}

Albores, Beatriz. 1995. Tules y sirenas: el impacto ecológico y cultural de la industrialización en el alto Lerma. Toluca: El Colegio Mexiquense y Gobierno del Estado de México.

Altieri, Miguel y Parviz Koohafkant. 2010. Globally Important Agricultural Heritage Systems: A Legacy for the Future. Roma: Food and Agriculture Organization (FAO).

Ávila, Patricia. 2007. El manejo del agua en territorios indígenas en México. Vol. 4 de la Serie de Agua. Ciudad de México: Banco Internacional de Reconstrucción y Fomento; Banco Mundial. Consultado el 20 de agosto del 2018. http://documentos.bancomundial.org/curated/es/2007/02/7514997/el-manejo-del-agua-en-territorios-indigenas-en-mexico.

—. 2014. Evaluación social regional sistema de microcuencas prioritarias "Cutzamala-La Marquesa”. Ciudad de México: CIEco; UNAM-Conafort-Semarnat.

Babidge, Sally. 2016. "Contested Value and an Ethics of Resources: Water, Mining and Indigenous People in the Atacama Desert Chile”. Australian Journal of Anthropology 27 (1): 84103. https://doi.org/10.1111/taja.12139.

Blaser, Mario. 2009. "Political Ontology. Cultural Studies without 'Cultures'?”. Cultural Studies 23 (5): 873-896. https://doi.org/10.1080/09502380903208023.

-. 2014. "Ontology and Indigeneity: On the Political Ontology of Heterogenous Assemblages". Cultural Geographies 21 (1): 49-58. https://doi.org/10.1177/1474474012462534.

Boelens, Rutgerd. 2014. "Cultural Politics and the Hydrosocial Cycle: Water, Power and Identity in the Andean Highlands”. Geoforum 57: 234-247. https://doi.org/10.1016/j.geoforum.2013.02.008.

Bonfil, Guillermo. 1987. El México profundo, una civilización negada. Ciudad de México: Grijalbo.

Budds, Jessica. 2010. "Relaciones sociales de poder y la producción de paisajes hídricos”. En Justicia hídrica: acumulación, conflicto y acción social, editado por Rutgerd Boelens, Leontien Cremers y Margreet Zwarteveen, 59-69. Lima: Instituto de Estudios Peruanos.

Comisión Nacional del Agua (Conagua). 1997. Diagnóstico ambiental de las etapas I, II y III del Sistema Cutzamala. Ciudad de México: Comisión Nacional del Agua.

-. 2005. Sistema Cutzamala. Agua para millones de mexicanos. Ciudad de México: Comisión Nacional del Agua; Secretaría del Medio Ambiente y Recursos Naturales.

Descola, Philippe. 2012. Más allá de naturaleza y cultura. Buenos Aires: Amorrortu.

Giménez, Gilberto. 2007. Estudios sobre la cultura y las identidades sociales. Ciudad de México: Conaculta-Iteso.

Gómez-Fuentes, Anahí Copitzy. 2009. "Un ejército de mujeres. Un ejército por el agua. Las mujeres indígenas mazahuas en México”. Agricultura, Sociedad y Desarrollo 6 (3): 207-221.

—. 2014. "Estado y política hidráulica en México: el conflicto de los indígenas mazahuas”. Agua y Territorio 4: 84-95. 
Ingold, Tim. 1993. "The Temporality of the Landscape”. World Archaeology 25 (2): 152-174.

Karpouzoglou, Timothy y Sumit Vij. 2017. "Waterscape: A Perspective for Understanding the Contested Geography of Water”. Wiley Interdisciplinary Reviews: Water 4 (3): e1210. DOI: $10.1002 /$ wat2.1210.

Legorreta, Jorge. 2006. El agua y la Ciudad de México: de Tenochtitlán a la megalópolis del siglo XXI. Ciudad de México: Universidad Autónoma Metropolitana.

Linton, Jamie y Jessica Budds. 2014. "The Hydrosocial Cycle: Defining and Mobilizing a Relational-Dialectical Approach to Water”. Geoforum 57: 170-180. https://doi.org/10.1016/j. geoforum.2013.10.008.

Maruri, María Elena. 2001. "Simbolismo acuático y cosmovisión en las prácticas religiosas. Una interpretación del modo de vida lacustre como pervivencia cultural en San Antonio La Isla, Estado de México”. Tesis de Maestría en Antropología Social, Centro de Investigaciones y Estudios Superiores en Antropología Social, Ciudad de México.

McLean, Jessica. 2017. "Water Cultures as Assemblages: Indigenous, Neoliberal, Colonial Water Cultures in Northern Australia”. Journal of Rural Studies 52: 81-89. https://doi. org/10.1016/j.jrurstud.2017.02.015.

Oehmichen, Cristina. 2002. "Comunidad y cosmovisión entre los mazahuas radicados en la Ciudad de México”. En Otopames, Memoria del Primer Coloquio, 123-148. Ciudad de México: Instituto de Investigaciones Antropológicas, Universidad Nacional Autónoma de México.

Pérez, Edgardo. 2006. "La naturaleza como percepción cultural. Montes y selvas en el Nuevo Reino de Granada, siglo XVIII”. Fronteras de la Historia 11: 57-84.

Romero-Contreras, Tonatiuh. 2005. "El uso y manejo del agua para la agricultura en el antiguo Valle de Toluca”. Páramo del Campo y la Ciudad 3 (7): 57-78.

Ruiz, Nazhelli y Javier Delgado Campos. 2013. "La cultura lacustre del alto Lerma: los vínculos perdidos entre patrimonio cultural y bienestar”. En Identidad y patrimonio cultural en América Latina, editado por Hernán Salas Quintanal, Mari Carmen Serra Puche e Íñigo González de la Fuente, 93-116. Ciudad de México: Instituto de Investigaciones Antropológicas, Universidad Nacional Autónoma de México.

Ruiz-Serna, Daniel y Carlos del Cairo. 2016. "Los debates del giro ontológico en torno al naturalismo moderno”. Revista de Estudios Sociales 55: 193-204.

Swyngedouw, Erik. 1995. "The Contradictions of Urban Water Provision: A Study of Guayaquil, Ecuador”. Third World Planning Review 17 (4): 387-405. DOI: 10.3828 / twpr.17.4.c8280 66008888322 .

- 1997. "Power, Nature and the City: The Conquest of Water and the Political Ecology of Urbanization in Guayaquil, Ecuador: 1880-1990". Environment and Planning A 29 (2): 311-332. https://doi.org/10.1068/a290311.

-. 1999. "Modernity and Hybridity: Nature, Regeneracionismo, and the Production of the Spanish Waterscape, 1890-1930". Annals of the Association of American Geographers 89 (3): 443-465.

-. 2004. Social Power and the Urbanization of Water: Flows of Power. Oxford: Oxford University Press. 
Tirel, Magali. 2013. Parteaguas. La propuesta mazahua a la Ciudad de México: reciprocidad, no-violencia y sustentabilidad. Ciudad de México: El Colegio de Michoacán; Universidad Nacional Autónoma de México.

Trejo, José Antonio y Emilio Gerardo Arriaga Álvarez. 2009. "Memoria colectiva: vida lacustre y reserva simbólica en el Valle de Toluca, Estado de México”. Convergencia 16 (50): 303-321.

Vizcarra-Bordi, Ivonne. 2001. "Y la lucha sigue entre sombreros y rebozos. Historia de una tierra de subsistencia marginal mazahua”. Estudios Agrarios 7 (18): 9-73.

-. 2002. Entre el taco mazahua y el mundo: la comida de las relaciones de poder, resistencia e identidades. Estado de México: Universidad Autónoma del Estado de México.

Worster, Donald. 2008. "El agua en la historia moderna. Temas y preocupaciones”. En Transformaciones de la tierra, editado por Donald Worster, 163-176. Montevideo: Coscoroba.

Yates, Julian, Leila Harris y Nicole Wilson. 2017. "Multiple Ontologies of Water: Politics, Conflict and Implications for Governance”. Environment and Planning D: Society and Space 35 (5): 797-815. DOI: https://doi.org/10.1177/0263775817700395. 